\title{
Chapter 3
}

\section{Modeling Cardiac Mechanics on a Sub-Cellular Scale}

\author{
Åshild Telle ${ }^{1}$, Samuel T. Wall ${ }^{1}$ and Joakim Sundnes ${ }^{1}$
}

\begin{abstract}
We aim to extend existing models of single-cell mechanics to the EMI framework, to define spatially resolved mechanical models of cardiac myocytes embedded in a passive extracellular space. The models introduced here will be pure mechanics models employing fairly simple constitutive laws for active and passive mechanics. Future extensions of the models may include a coupling to the electrophysiology and electro-diffusion models described in the other chapters, to study the impact of spatially heterogeneous ion concentrations on the cell and tissue mechanics.
\end{abstract}

\subsection{Introduction}

A vast range of models have been developed for the force development of cardiac and skeletal muscle, on the scale of a single cross bridge (10), myofilament (3), sarcomere (2), and the complete cell (13). The scales involved and the main functional units considered on each scale are schematically illustrated in Figure 3.1. Common to most existing models is the fact that they focus on a single spatial scale, and any coupling between scales is fairly crudely represented. As an example, the model by Rice et al. (13) is essentially a model of a single sarcomere (Fig. 3.1 D), which is normalized and then scaled to yield a realistic force output for cell- and tissue-level mechanics applications. Other models provide detailed descriptions of mechanisms and interactions on a molecular level (Fig. 3.1 F)(4; 3), and are able to capture many of the characteristic non-linearities of muscle cell mechanics. However, key aspects of mechanical activation and force-length relationships are still not fully understood, and they may be the result of interactions between individual sarcomeres and myofibril bundles. A few attempts have been made at modeling interactions at this scale, and

${ }^{1}$ Simula Research Laboratory, Norway 
A

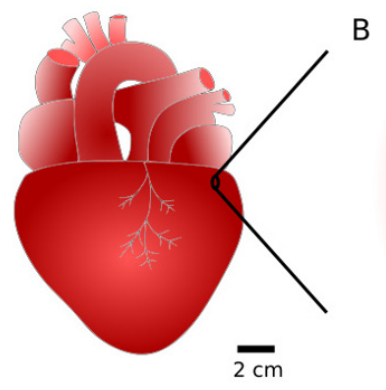

B

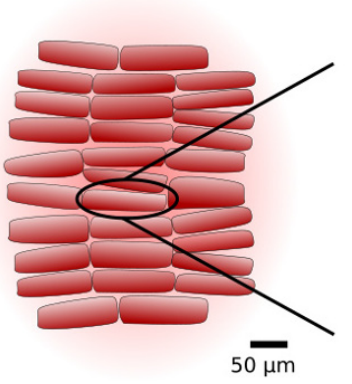

$\mathrm{C}$

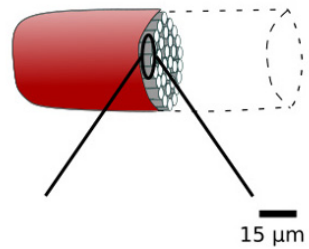

$15 \mu \mathrm{m}$
$\mathrm{F}$

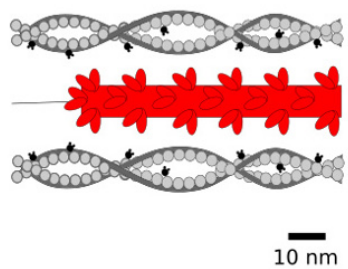

E

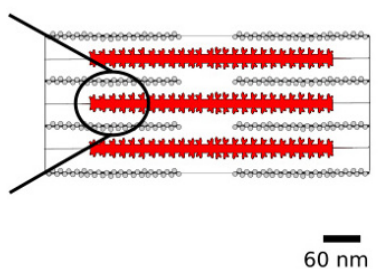

D

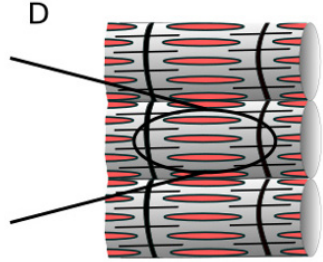

$\overline{0.5 \mu \mathrm{m}}$

Fig. 3.1: The heart (A) is mainly composed of cardiac muscle cells, also called cardiomyocytes (B). Each cell (C) is composed of long tubes of sarcomeres (D), in which the thin and thick myofilaments overlap in layers $(\mathrm{E})$. The interaction between these $(\mathrm{F})$ causes the cardiac muscle to contract in a process called the cross-bridge cycle.

have shown potentially interesting emergent behaviours $(2 ; 11)$. Furthermore, heart failure and other pathologies are linked with heterogeneous intracellular calcium concentration resulting from disruptions of the calcium regulation system. Describing the effect of such heterogeneities on the cell contraction and force development requires spatially resolved mechanics models on the sub-cellular scale.

Finite element models of contracting myocytes have been proposed (8; 14), and have been used to explore the impact of model assumptions, calcium heterogeneity, and boundary conditions. The model presented by Ruiz-Baier et al. (14) describes the individual myocyte as a hyperelastic material, and uses an active strain approach to describe the contraction. Both the passive and active mechanical properties are assumed to be homogeneous, but sub-cellular heterogeneities can easily be introduced. We here propose to extend the single myocyte model in (14) to include the extracellular domain, and to model collections of cells, based on similar ideas used for the electrophysiology model presented in (17, 18) and (7, Chapter 1). 


\subsection{Models and Methods}

The motion and deformation of the heart can be described by the classical theory of non-linear solid mechanics. The primary unknown in our computational model will be the displacement vector $u$, which for each material point describes the difference between its current and original position. We have $u=x-X$, where $X$ is the original (reference) position of a point, and $x$ is its position after the deformation. From the displacement vector we can define the deformation gradient $F=\partial x / \partial X=$ $I+\partial u / \partial X$, which is an essential quantity describing the deformation of a solid. See for instance (6) for a detailed introduction to non-linear solid mechanics.

A characteristic feature of the heart and other muscles is that they contract and deform even in the absence of external loads. The overall deformation and mechanical state of the heart depends both on this active contraction and on the passive mechanical properties of the tissue. There are two main approaches for modeling the coupling of active and passive mechanics in cardiac tissue, often referred to as active strain and active stress. Both approaches are based on modeling the active and passive contributions separately, then combining them into a complete coupled model.

In the active strain approach, the active-passive coupling is incorporated through a multiplicative decomposition of the deformation gradient $F$ into active and passive components, $F=F_{p} F_{a}$. Here, $F_{a}$ represents an active deformation governed by the cell state, and $F_{p}$ is a passive elastic deformation which ensures compatibility with loads and kinematic boundary conditions. The active stress approach is based on an additive split of the stress tensor into its active and passive components. In terms of the first Piola-Kirchhoff stress tensor $P$, the stress is written as $P=P_{p}+P_{a}$, where $P_{a}$ is a function of the cellular activation state and $P_{p}$ is a standard elastic stress derived from a strain energy function.

Both of these approaches have their strengths and weaknesses. In general, the active strain approach is considered to be more suitable for deriving mathematically wellbehaved constitutive laws, while the active stress concept is more easily coupled to biophysically detailed models of cell contraction.

\subsubsection{Fundamental Equations}

In this study we will primarily use the active stress approach, but for completeness we also present the equations arising from the active strain approach. This model can be derived as a direct extension of the single myocyte model in (14), using a similar approach as in $(17,18)$ to consider both the intra- and extracellular domains: 


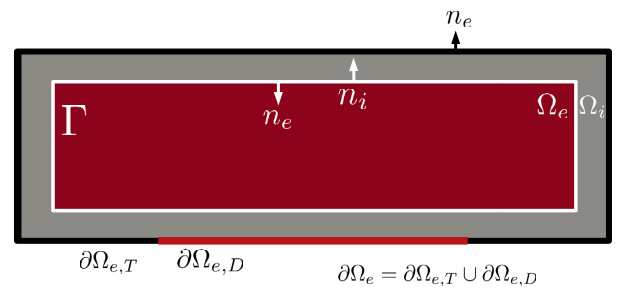

Fig. 3.2: Illustration of the intra- and extracellular domains for a single cell and its surroundings.

$$
\begin{array}{lll}
a: \nabla \cdot P_{i}=0, & b: P_{i}=\frac{\partial \Psi_{i}}{\partial F_{j}}, \quad c: F_{i}=F_{i}^{p} F_{i}^{a}, & \text { in } \Omega_{\mathrm{i}}, \\
d: \nabla \cdot P_{e}=0, & e: P_{e}=\frac{\partial \Psi_{e}}{\partial F_{e}}, & \text { in } \Omega_{\mathrm{e}}, \\
f: u_{i}=u_{e}, & g: n_{i} \cdot P_{i}=n_{e} \cdot P_{e}, & \text { on } \Gamma, \\
h: n_{e} \cdot P_{e}=0, & & \text { on } \partial \Omega_{\mathrm{e}, \mathrm{T}}, \\
i: u=0, & & \text { on } \partial \Omega_{\mathrm{e}, \mathrm{D}}
\end{array}
$$

Here, $\Omega_{i}$ and $\Omega_{e}$ are the intra- and extracellular domains, respectively, $\Gamma$ is the interface between the domains, with the normal vector $n_{i}$ pointing out of the intracellular domain and $n_{e}$ out of the extracellular domain. Finally, $\partial \Omega_{e, T}$ and $\partial \Omega_{e, D}$ are the parts of the outer boundary $\partial \Omega_{e}$ subject to traction- and displacement boundary conditions, respectively. See Figure 3.2 for a sketch of a typical computational domain, including a single cell and its immediate surroundings. Following (14), we here apply the active strain approach to incorporate active contraction of the myocyte, where the intracellular deformation gradient $F_{i}$ is decomposed as described above. The passive part is assumed to be hyper-elastic and derived from a strain energy function, see for instance (6) for details. A common choice for the active part is $F_{i}^{a}=\operatorname{diag}\left((1-\gamma),(1-\gamma)^{-1 / 2},(1-\gamma)^{-1 / 2}\right)$, where $\gamma$ describes the fiber contraction and is a function of the cell activation state. For a more detailed introduction and discussion of active strain models, we refer to (1).

The active stress model is the most widely used approach for modeling coupled active and passive mechanics on tissue level, and this is the approach we will employ in the subsequent numerical experiments. In the present context the active stress model involves a decomposition of the intracellular first Piola-Kirchhoff stress $P_{i}$ into a passive elastic part $P_{i}^{p}$ and an active part $P_{i}^{a}$. The passive stress is derived from a strain energy function in the usual way, while the active stress is a function of the cell activation state. For the simplified model considered here we write the active stress as a function of time and the local fiber stretch $\lambda$, but the approach can easily be extended to include detailed biophysical models of the contractile mechanisms. The full active stress model may be written as 


$$
\begin{array}{lll}
a: \nabla \cdot P_{i}=0, & b: P_{i}=\frac{\partial \Psi_{i}}{\partial F_{F}}+P_{i}^{a}(t, \lambda), & \text { in } \Omega_{\mathrm{i}}, \\
c: \nabla \cdot P_{e}=0, & d: P_{e}=\frac{\partial \Psi_{e}}{\partial F_{e}}, & \text { in } \Omega_{\mathrm{e}}, \\
e: u_{i}=u_{e}, & f: n_{i} \cdot P_{i}=n_{e} \cdot P_{e}, & \text { on } \Gamma, \\
g: n_{e} \cdot P_{e}=0 & & \text { on } \partial \Omega_{\mathrm{e}, \mathrm{T}}, \\
h: u=0 & & \text { on } \partial \Omega_{\mathrm{e}, \mathrm{D}}
\end{array}
$$

Both approaches treat the extracellular domain in the same way, as a passive hyperelastic material governed by a strain energy function $\Psi_{e}$. As given by (3.1) f-g and (3.2) g-h we assume continuity of stresses $P_{i}, P_{e}$ and displacements $u_{i}, u_{e}$ across the cell membrane $\Gamma$, implying that the membrane itself has no stiffness. The outer boundary $\Omega_{e}$ is assumed to be stress free, with Dirichlet conditions applied to parts of the boundary to avoid rigid body motion. Models for the active stress $P_{i}^{a}$ come in many forms, including simple phenomenological models as well as detailed biophysical models of cell electro-mechanics $(12 ; 13)$. For the present study we apply a simple model where the active stress is derived from a (pseudo-) strain energy in the same way as the passive stress:

$$
P_{i}^{a}=\frac{\partial \Psi_{i}^{a}}{\partial F} .
$$

Here, $\Psi_{i}^{a}$ is given by

$$
\Psi_{i}^{a}=\frac{T_{\text {active }}(t)}{2} \lambda^{2},
$$

where $\lambda=\left\|F e_{1}\right\|$ is the stretch in the so-called fiber direction (i.e. the main orientation of the muscle cells), defined by the unit vector $e_{1}$, and $T_{\text {active }}(t)$ is a prescribed function defining the active contractile force as a function of time.

\subsubsection{Specific Model Choices}

In this section we describe specific choices of the constitutive laws describing active and passive material properties in the models above, to arrive at a complete model that can be solved for the deformations and stresses. As noted above, we will in the following only consider the active stress model, given by (3.2). For the strain energy defining the passive stress-strain relationships we have applied a model from (19), which belongs to the family of models first presented by Guccione et al. (5). The same form of strain energy is used in the intra- and extracellular domains, but we allow the material parameters to be different. Both domains are modeled as nearly incompressible, with volume changes during deformations controlled by a penalty term. We have

$$
\begin{array}{ll}
\Psi_{i}=C_{i}\left(e^{Q_{i}}-1\right)+\kappa(J \ln J-J+1) & x \in \Omega_{i}, \\
\Psi_{e}=C_{e}\left(e^{Q_{e}}-1\right)+\kappa(J \ln J-J+1) & x \in \Omega_{e},
\end{array}
$$


where $Q_{i}, Q_{e}$ are functions depending on components of the Green-Lagrange strain tensor $E=\frac{1}{2}\left(F^{T} F-I\right)$ :

$$
\begin{array}{r}
Q_{j}=b_{f, j} E_{11}^{2}+b_{t, j}\left(E_{22}^{2}+E_{33}^{2}+E_{23}^{2}+E_{32}^{2}\right) \\
+b_{f s, j}\left(E_{12}^{2}+E_{21}^{2}+E_{13}^{2}+E_{31}^{2}\right) .
\end{array}
$$

Furthermore $C_{j}, b_{f, j}, b_{t, j}$, and $b_{f s, j}$, for $j=i, e$ are material parameters characterizing the material's stiffness to the various strain modes, $k$ is a penalty parameter that controls the volume changes, and $J=\operatorname{det} F$. For a fully incompressible deformation we have $J=1$, and in our nearly incompressible model we tune the parameter $\kappa$ to keep $J \approx 1$.

In its most general form, the materials described by (3.4)-(3.6) are are transversely isotropic, which is a special case of orhtotropic materials. While an orthotropic material has different mechanical properties in three different directions, a transversely isotropic material is isotropic in planes normal to a characteristic direction. Passive cardiac tissue is known to behave as an orthortopic material (9), with the three directions dictated by the orientation and organization of the myocytes. However, a transversely isotropic material is shown to be a good approximation, with material isotropy in planes normal to the fiber direction, the main orientation of the muscle cells. The details of the intra- and extracellular material behavior in our micro-structural model are less well-studied, and the degree of anisotropy has not been characterized. From the microstructure of the contractile apparatus occupying most of the intracellular space (see Figure 3.1) it is natural to assume anisotropic behavior, but the exact degree of aniostropy is not known. As a starting point, we set the intracellular material parameters to

$$
b_{f, i}=8, \quad b_{t, e}=2, \quad b_{f s, e}=4 .
$$

For the extracellular space we assume isotropic material behaviour, setting

$$
b_{f, e}=b_{t, e}=b_{f s, e}=1 .
$$

The bulk compressibility was set to $\kappa=1000 \mathrm{kPa}$ in both domains, while we explored different values of the scaling parameters $C_{i}$ and $C_{e}$, to be specified below.

For the active stress model defined in (3.3) we have used a pre-computed transient tension $T_{\text {active }}(t)$ as shown in Figure 3.3. The curve was computed using the model of Rice et al. (13) with default parameters, which outputs a normalized force. This value was then scaled such that the peak value reaches $2 \mathrm{kPa}$, giving a reasonable contractile stress for our application. 

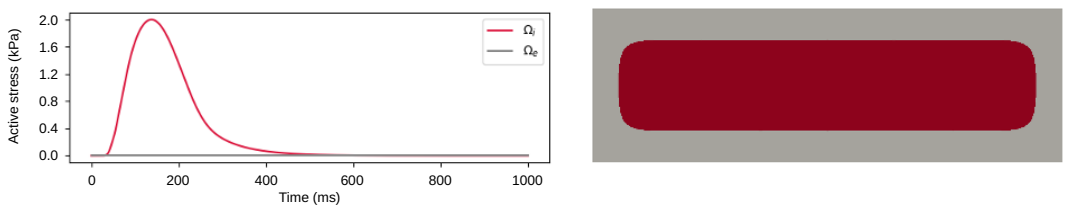

Fig. 3.3: Transient tension $T_{\text {active }}(t)$ over time (left), first computed in (13), then scaled to give values on a reasonable scale. In the intracellular domain the active tension is homogeneously set to this value; in the extracellular domain there is no such tension, implemented as being set to zero for all time steps.

\subsubsection{Numerical Methods}

The problem defined by (3.2) is solved with the displacement $u$ as the primary unknown. To solve the system with the finite element method, it is convenient to formulate it as a single PDE defined over the entire domain $\Omega=\Omega_{i} \cup \Omega_{e}$. Such a formulation is not possible for the strong form of the PDEs, so we first need to derive the weak form of the equations. Starting with (3.2)a, we define a suitable vector function space $V\left(\Omega_{i}\right)$ defined over the intracellular domain, multiply the equation with a test function $v \in V\left(\Omega_{i}\right)$ and integrate by parts, to arrive at a weak formulation

$$
\int_{\Omega_{i}} P_{i} \cdot \nabla v d x-\int_{\Gamma}\left(n_{i} \cdot P_{i}\right) v=0 .
$$

This equation is to be satisfied for all $v \in V\left(\Omega_{i}\right)$. Performing the same steps for the extracellular domain, and using the boundary condition (3.2)g on the outer boundary, we get

$$
\int_{\Omega_{e}} P_{e} \cdot \nabla v d x-\int_{\Gamma}\left(n_{e} \cdot P_{e}\right) v=0 .
$$

This equation should hold for all test functions $v \in V\left(\Omega_{e}\right)$, where $V\left(\Omega_{e}\right)$ is a suitable space of functions defined over the domain $\Omega_{e}$. Using similar arguments as in (15), we can define a function space $V(\Omega)$ as the set of functions defined over $\Omega$ that belong to both $V\left(\Omega_{i}\right)$ and $V\left(\Omega_{e}\right)$ and are continuous over $\Gamma$. With this definition, we may add the two weak forms above to obtain

$$
\int_{\Omega_{i}} P_{i} \cdot \nabla v d x-\int_{\Gamma}\left(n_{i} \cdot P_{i}\right) v+\int_{\Omega_{e}} P_{e} \cdot \nabla v d x-\int_{\Gamma}\left(n_{e} \cdot P_{e}\right) v=0 .
$$

Which is to be satisfied for all $v \in V(\Omega)$. Since $n_{e}=-n_{i}$, the surface integrals over $\Gamma$ cancel because of (3.2)f. We can also use (3.2)e to define a single displacement field over $\Omega$, and we are left with the following weak form: Find $u \in V(\Omega)$ such that 
A

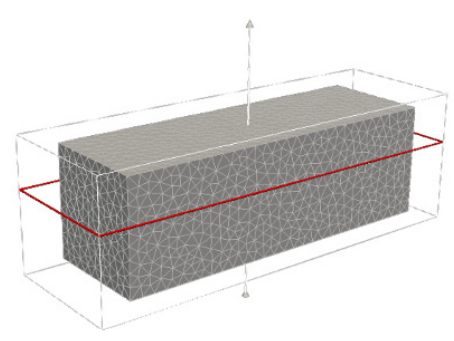

C

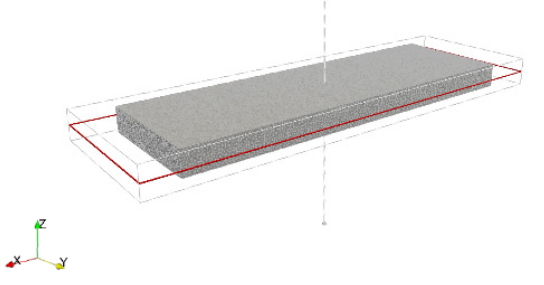

B

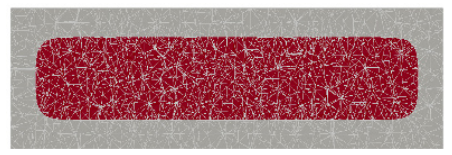

D

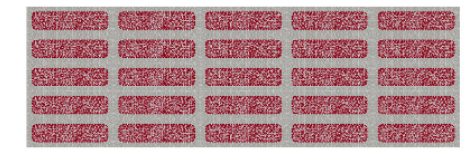

$\Delta$

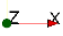

Fig. 3.4: A: Volume element of one single cell; lines indicate cross section area. B: Cross section along longitudial direction of the cell. C: Volume element, 5 x 5 cells; lines indicate cross section area. D: Cross section along longitudial direction of the cells.

$$
\int_{\Omega} P \cdot \nabla v d x=0
$$

is satisfied for all $v \in V(\Omega)$. with $P$ defined by (3.2)b and (3.2)d in the respective domains.

\subsection{Results}

We here present a number of numerical experiments to illustrate the general behavior of the models defined above. The code is implemented using FEniCS, and an archieved version of the code is available, see (16).

For the simulations we used two different meshes; one representing a single cell and one representing a sheet of five by five cells, see Figure 3.4. Both meshes include subdomains defining the intra- and extracellular domains, separated by the cell membrane. To avoid rigid body motion, we keep a few points in the middle fixed. The rest of the boundary is kept unloaded to allow free contraction of the cells. 
For each experiment we calculated the Green-Lagrange strain tensor $E$ and the Cauchy stress tensor $\sigma$, given by

$$
\sigma= \begin{cases}|F|^{-1} P_{i} F^{T} & x \in \Omega_{i} \\ |F|^{-1} P_{e} F^{T} & \text { otherwise }\end{cases}
$$

On matrix form we can can write these out as

$$
E=\left[\begin{array}{lll}
E_{11} & E_{12} & E_{13} \\
E_{21} & E_{22} & E_{23} \\
E_{31} & E_{32} & E_{33}
\end{array}\right] \quad \sigma=\left[\begin{array}{lll}
\sigma_{11} & \sigma_{12} & \sigma_{13} \\
\sigma_{21} & \sigma_{22} & \sigma_{23} \\
\sigma_{31} & \sigma_{32} & \sigma_{33}
\end{array}\right]
$$

and for each of these we present plots for the first and the middle components, $\left(E_{11}, E_{22}, \sigma_{11}, \sigma_{22}\right)$, which characterize strain and stress in the fiber and cross-fiber directions.

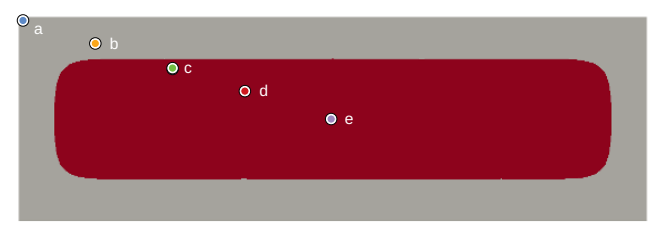

Fig. 3.5: Tracking points, for which we evaluate functions of interest across various experiments. The points are uniformly distributed on a line from one corner to the middle, in the xy-direction, corresponding to the cross-section shown in Figure 3.4. Two of them are both located in the extracellular subdomain, and one should expect them to show different patterns than the three located in the intracellular subdomain.

We first considered a single cell, and simulated contraction over a single cardiac cycle with homogeneous active force applied throughout the cell. For this simulation we chose parameter values $C_{e}=C_{i}=0.5$. The results are presented in Figure 3.6, where we observe that the deformation follows the expected pattern of a contraction in the longitudinal direction of the cell. Furthermore, in spite of the homogeneous applied active stress we see slight spatial variations in the deformation state, resulting from the discontinuity of active force across the cell membrane.

Similar patterns are observed in the simulation of the sheet of 25 cells, shown in Figure 3.7. In this experiment the same active stress transient through the intracellular domain of all the cells, with the same material parameters. We still observe spatial variations in the deformation pattern - each cells is affected by mechanical deformation around them.

We then considered two cases where we kept all parameters but one fixed, exploring the choices of material stiffness parameters $C_{e}$ and $C_{i}$. The results are presnted in 

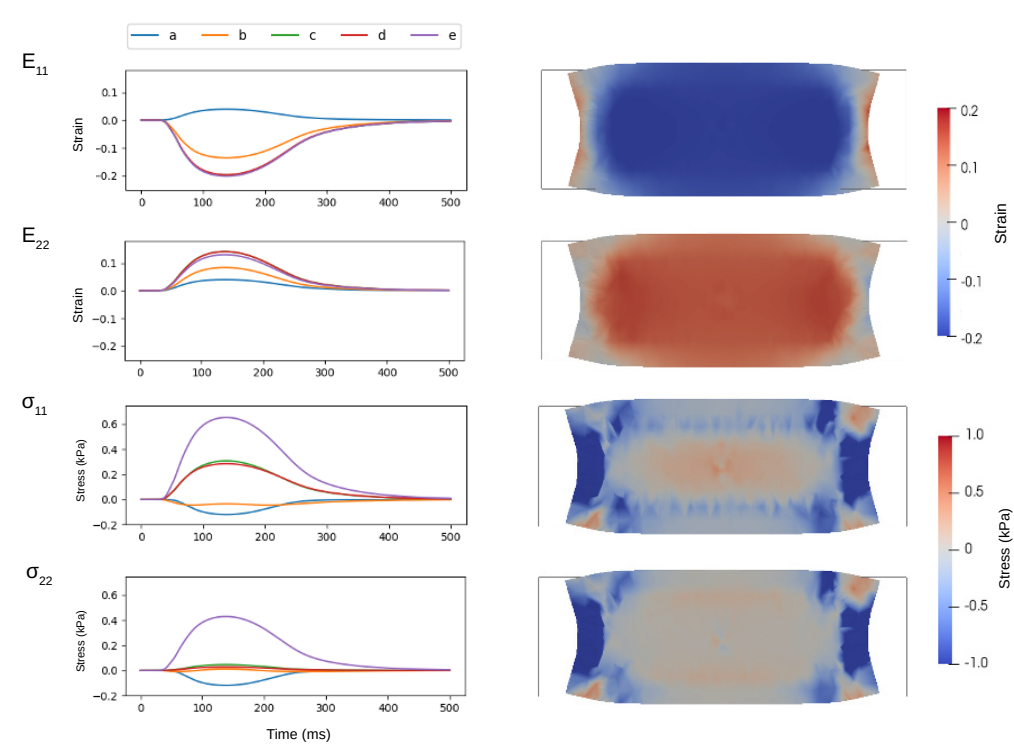

Fig. 3.6: First and middle components of the Cauchy stress tensor $\sigma$ and GreenLagrange strain $E$, for a single cell. The plots to the left shows values plotted over time, for the first $500 \mathrm{~ms}$ (out of 1000), following tracking points as shown in Figure 3.5. The plots to the right shows values over a cross-section as shown in Figure 3.4 , as the active tension reaches it's peak value. The grey rectangle indicates initial configuration.

Figures 3.8 and 3.9. These simulations were again performed on a mesh representing a single cell, with active force applied as described above. For the first experiment we kept $C_{e}$ fixed at 0.5 , changing $C_{i}$; that is, we let the material stiffness in the extracellular domain remain the same while increasing the stress/strain scaling parameter in the intracellular domain. As $C_{i}$ increases the material becomes stiffer, and for the same active stress applied, one should expect less contraction. This can indeed be observed; both components of the Cauchy stress tensor (in magnitude) and the strain tensor decreases everywhere, and for the last three parameter choices there is almost no difference in deformation. On the other hand, we still apply an active stress in the intracellular domain, and we observe that the strain close to the membrane doesn't change much even if it changes everywhere else.

For the next experiment we changed to keeping $C_{i}=0.5$ constant, while increasing $C_{e}$. We can observe higher Cauchy stress for the first component, and lower Cauchy stress for the second component, with increasing values of $C_{e}$. The strain decreases for both components. This is exactly as expected - in one end of the spectrum, having 

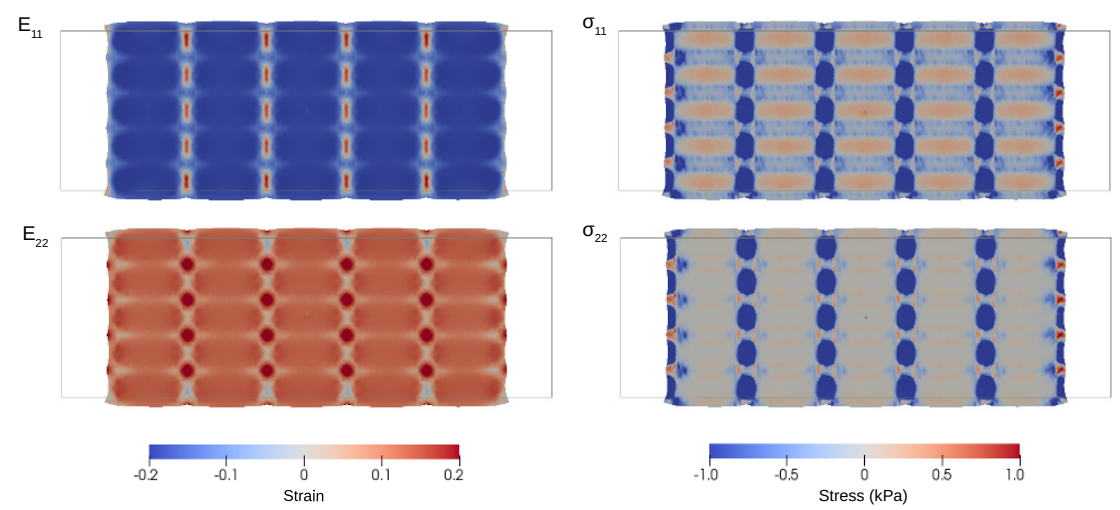

Fig. 3.7: First and middle components of the Cauchy stress tensor $\sigma$, and and GreenLagrange strain $E$, for 5 x 5 cells. Values are plotted over the cross-section as shown in 3.4 , as the active tension reaches it's peak value. The grey rectangle indicates initial configuration.

$C_{e}=0.5$, one would expect the extracellular subdomain to not affect the intracellular domain as it's rather flexible. For a given tension in the intracellular domain, it will just move along quite easily, while the overall behaviour in the whole domain is governed by the contraction inside the cell. As $C_{e}$ increases, the material is modeled as stiffer and hence constrain the movement more. For very high values the material is so stiff that it hardly moves, efficiently keeping the membrane close to fixed.

\subsection{Discussion}

We have presented a general framework for modeling cardiac mechanics on a subcellular scale, by extending a model of the type defined in (14) to the extracellular domain. A series of preliminary numerical experiments demonstrate that the model behaves as expected, with the discontinuity across the cell membrane giving rise to spatially varying deformation fields even though both the active stress and other model parameters are spatially homogeneous over the intracellular domain.

The main purpose of this work was to present the model framework and to illustrate the general behaviour of the model, while more detailed investigations and model extensions are left for future studies. A complete list of model limitations and potential extensions would be too extensive to present here, but it is worth commenting on a few of the most obvious ones. First, the model derivation above included a number of simplifying assumptions on the mechanical properties of the cell membrane and 
A

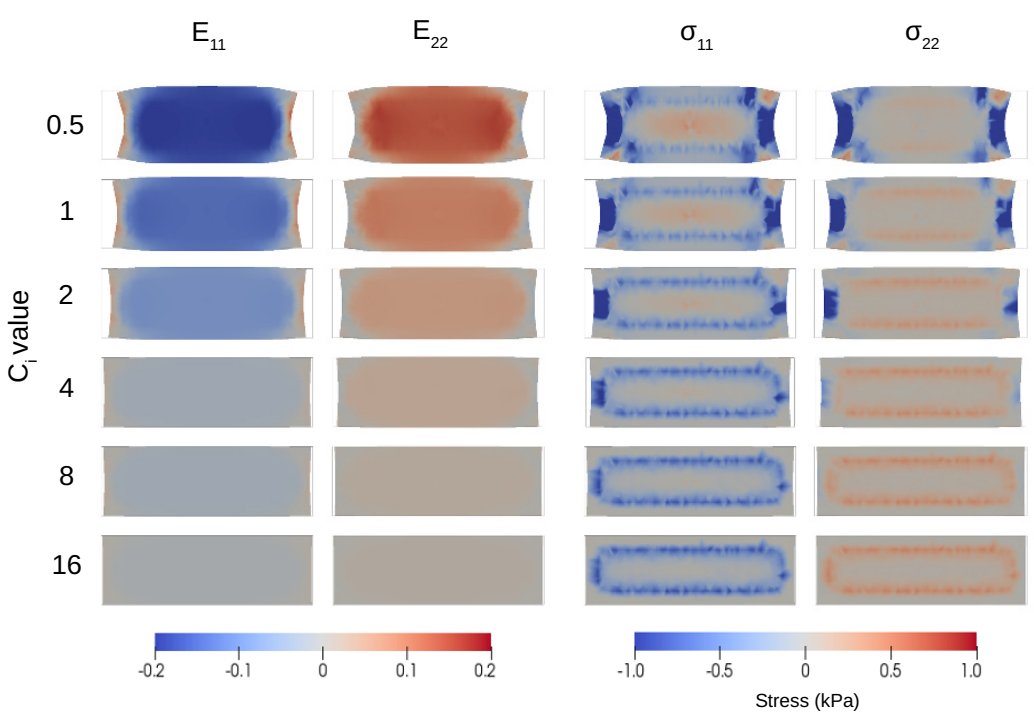

B
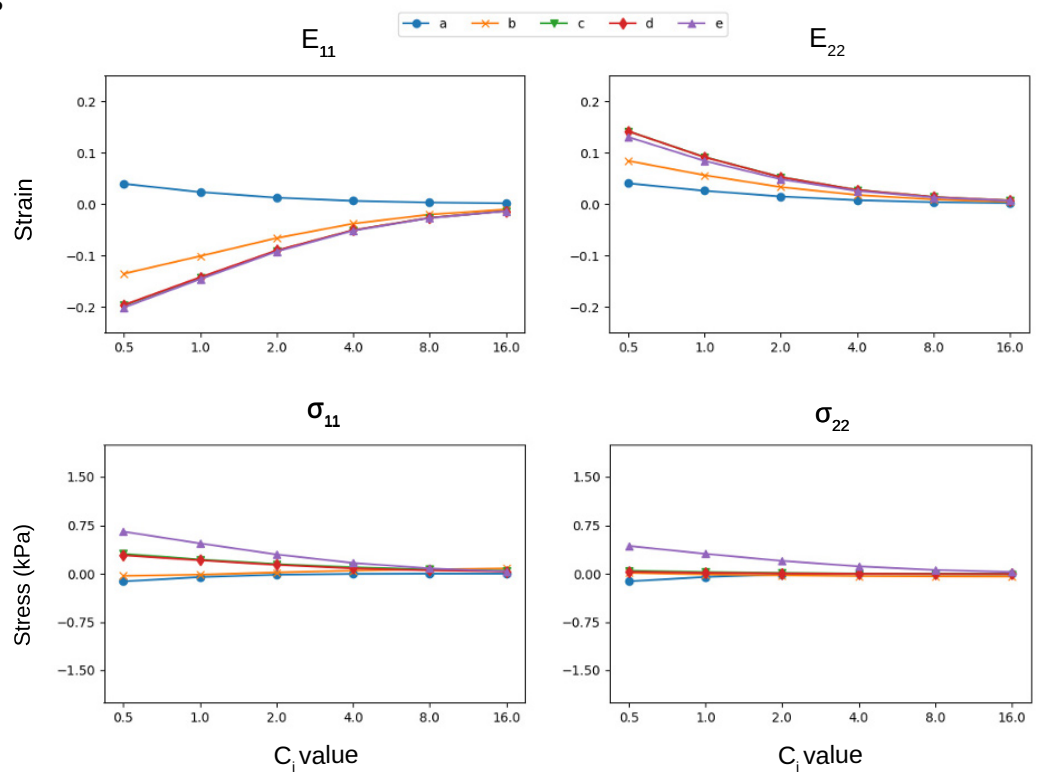

Fig. 3.8: First and middle components of the Cauchy stress tensor $\sigma$ and GreenLagrange strain $E$, for a single cell, as we vary the parameter $C_{i}$, which defines the stiffness of the material in the intracellular domain. Panel A shows spatial variation over a cross-section of the cell (see Figure 3.4), at peak. Panel B shows how the value, at peak, changes in given tracking points (see Figure 3.5). 
A

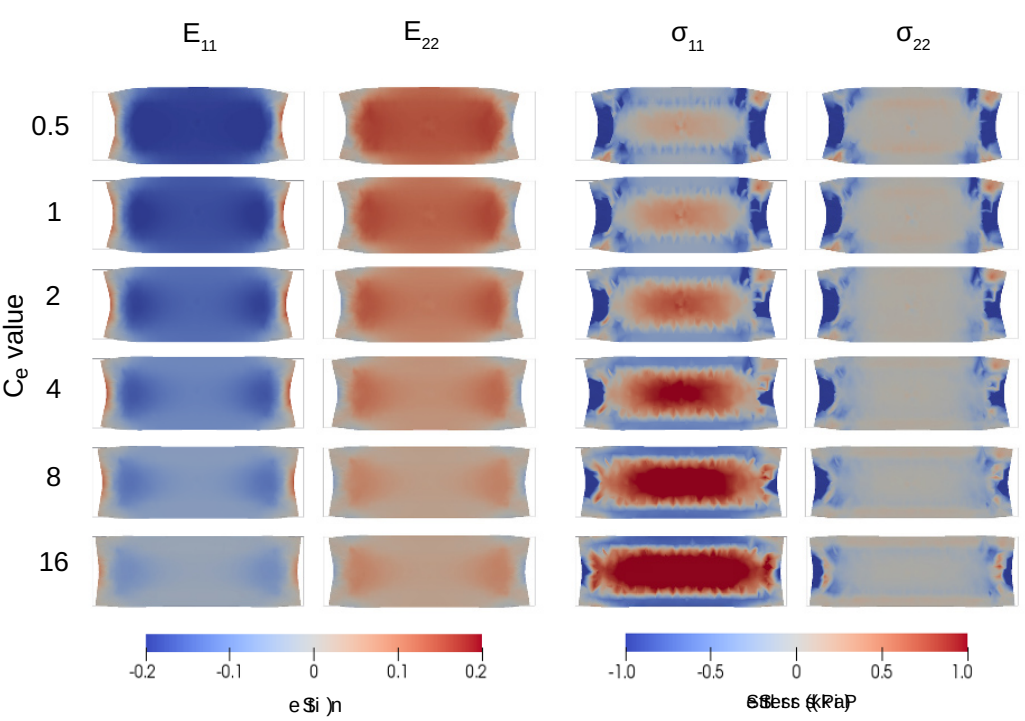

B
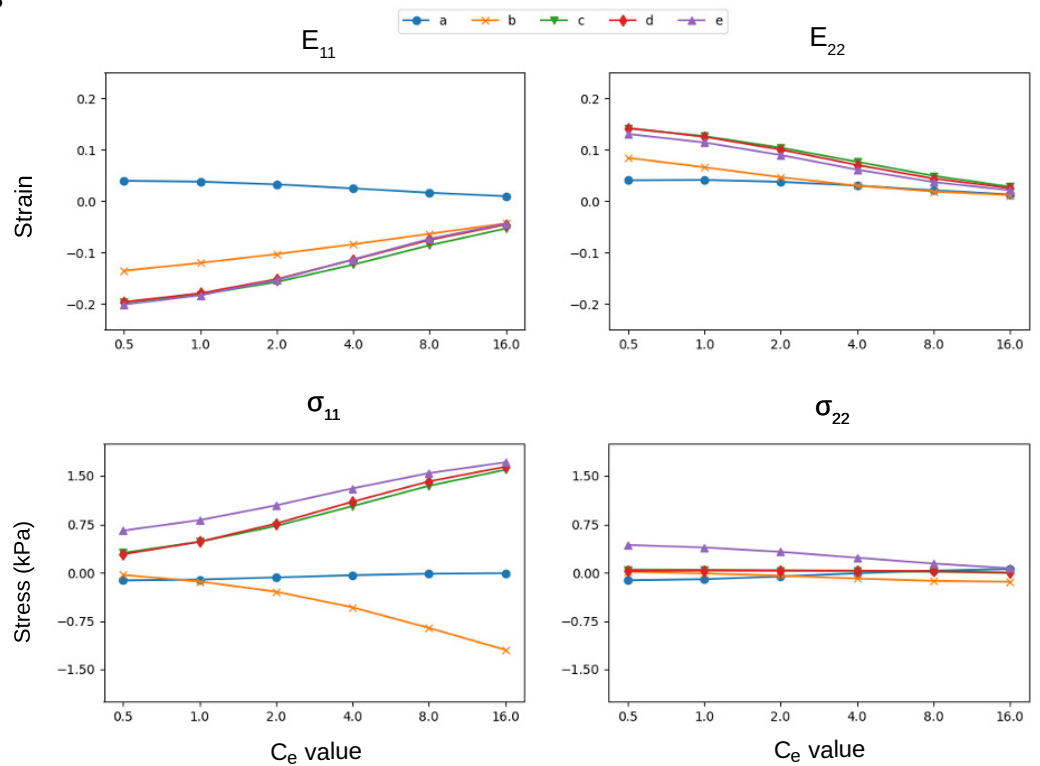

Fig. 3.9: First and middle components of the Cauchy stress tensor $\sigma$ and GreenLagrange strain $E$, for a single cell, as we vary the parameter $C_{e}$, which defines the stiffness of the material in the extracellular domain. Panel A shows spatial variation over a cross-section of the cell (see Figure 3.4), at peak. Panel B shows how the value, at peak, changes in given tracking points (see Figure 3.5). 
the two domains. The continuity of stress across the cell membrane implies that the membrane itself has no stiffness, which is obviously incorrect, but it may be a reasonable assumption for many applications. The impact of different membrane mechanical properties should be explored further in a future study. Similarly, both the intra- and extracellular domains are assumed to be hyperelastic materials, which is probably a fairly crude approximation of the actual behaviour. In reality both of these domains are complex compositions of fluids and various embedded proteins structures, and the material behavior is most likely quite complex. Visco-elastic material models could potentially be a more accurate description than the hyper-elastic models applied here, but the required level of detail and material model complexity remains to be determined. Finally, we have here assumed that both domains are initially in a stress-free resting state, while experiments have shown that the extracellular matrix shrinks considerably when the myocytes are removed. Thus indicates that the resting state is actually an equilibrium state with non-zero stress in both domains, and accurately capturing the overall mechanical behaviour may require including this pre-stress in the model.

In general, the level of detail and complexity of the model formulation will be dictated by the application. Some applications may require further development of the model along the lines suggested above, while for studies of a more qualitative nature the simplest version would be sufficient. One obvious application of the developed model framework, where a fairly simple model would probably give interesting results, is to study the impact of heterogeneities in calcium concentration and mechanical properties on the contractile properties of cells and tissue.

Acknowledgements The simulations were performed on resources provided by UNINETT Sigma2 - the National Infrastructure for High Performance Computing and Data Storage in Norway. The meshes used for the simulations were generated using software provided by Miroslav Kuchta.

Open Access This chapter is licensed under the terms of the Creative Commons Attribution 4.0 International License (http://creativecommons.org/licenses/ by/4.0/), which permits use, sharing, adaptation, distribution and reproduction in any medium or format, as long as you give appropriate credit to the original author(s) and the source, provide a link to the Creative Commons license and indicate if changes were made.

The images or other third party material in this chapter are included in the chapter's Creative Commons license, unless indicated otherwise in a credit line to the material. If material is not included in the chapter's Creative Commons license and your intended use is not permitted by statutory regulation or exceeds the permitted use, you will need to obtain permission directly from the copyright holder.

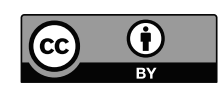




\section{References}

1. Ambrosi D, Pezzuto S (2012) Active stress vs. active strain in mechanobiology: constitutive issues. Journal of Elasticity 107(2):199-212

2. Campbell KS (2009) Interactions between connected half-sarcomeres produce emergent mechanical behavior in a mathematical model of muscle. PLoS computational biology 5(11):e1000560

3. Campbell SG, Lionetti FV, Campbell KS, McCulloch AD (2010) Coupling of adjacent tropomyosins enhances cross-bridge-mediated cooperative activation in a markov model of the cardiac thin filament. Biophysical journal 98(10):2254-2264

4. Chase PB, Macpherson JM, Daniel TL (2004) A spatially explicit nanomechanical model of the half-sarcomere: myofilament compliance affects ca 2+-activation. Annals of biomedical engineering 32(11):1559-1568

5. Guccione J, McCulloch A, Waldman L (1991) Passive material properties of intact ventricular myocardium determined from a cylindrical model. Journal of Biomechanical Engineering 113(1):42-55, DOI 10.1115/1.2894084

6. Holzapfel GA (2000) Nonlinear solid mechanics: a continuum approach for engineering. Wiley

7. Jæger KH, Tveito A (2020) Derivation of a cell-based mathematical model of excitable cells. In: Tveito A, Mardal KA, Rognes ME (eds) Modeling excitable tissue - The EMI framework, Simula Springer Notes in Computing, SpringerNature

8. Laadhari A, Ruiz-Baier R, Quarteroni A (2013) Fully eulerian finite element approximation of a fluid-structure interaction problem in cardiac cells. Int J Numer Meth Engng 96:712-738

9. LeGrice IJ, Smaill B, Chai L, Edgar S, Gavin J, Hunter PJ (1995) Laminar structure of the heart: ventricular myocyte arrangement and connective tissue architecture in the dog. American Journal of Physiology-Heart and Circulatory Physiology 269(2):H571-H582

10. Mijailovich SM, Nedic D, Svicevic M, Stojanovic B, Walklate J, Ujfalusi Z, Geeves MA (2017) Modeling the actin. myosin atpase cross-bridge cycle for skeletal and cardiac muscle myosin isoforms. Biophysical journal 112(5):984-996

11. Nakagome K, Sato K, Shintani SA, Ishiwata S (2016) Model simulation of the spoc wave in a bundle of striated myofibrils. Biophysics and physicobiology 13:217-226

12. Nash MP, Panfilov AV (2004) Electromechanical model of excitable tissue to study reentrant cardiac arrhythmias. Progress in Biophysics and Molecular Biology 85(2-3):501-522

13. Rice JJ, Wang F, Bers DM, de Tombe PP (2008) Approximate Model of Cooperative Activation and Crossbridge Cycling in Cardiac Muscle Using Ordinary Differential Equations. Biophysical Journal 95(5):2368-2390

14. Ruiz-Baier R, Gizzi A, Rossi S, Cherubinie C, Laadhari A, Filippi S, Quarterone A (2014) Mathematical modelling of active contraction in isolated cardiomyocytes. Mathematical medicine and biology 31:259-283 
15. Sundnes J, Lines GT, Cai X, Nielsen BF, Mardal KA, Tveito A (2007) Computing the electrical activity in the heart, vol 1 . Springer Science \& Business Media

16. Telle $\AA$ (2020) Software for EMI - Modeling cardiac mechanics on a sub-cellular scale. DOI 10.5281/zenodo.3702168, URL https://doi.org/10.5281/zenodo.3702168

17. Tveito A, Jæger KH, Lines GT, Paszkowski Ł, Sundnes J, Edwards AG, Māki-Marttunen T, Halnes G, Einevoll GT (2017) An evaluation of the accuracy of classical models for computing the membrane potential and extracellular potential for neurons. Frontiers in computational neuroscience 11:27

18. Tveito A, Jager KH, Kuchta M, Mardal KA, Rognes ME (2017) A cell-based framework for numerical modeling of electrical conduction in cardiac tissue. Frontiers in Physics 5:48, DOI 10.3389/fphy.2017.00048, URL https://www.frontiersin.org/article/ 10.3389/fphy.2017.00048

19. Usyk TP, LeGrice IJ, McCulloch AD (2002) Computational model of three-dimensional cardiac electromechanics. Computing and Visualization in Science 4(4):249-257 